\title{
Performance of Polar Codes over Generalized Correlated Fading Channels
}

\author{
Francisco Caio Parente de Barros, Flávia Camila Morais de Oliveira, Maria de Lourdes Melo Guedes Alcoforado \\ and Waslon Terllizzie Araújo Lopes
}

\begin{abstract}
Polar coding, as introduced by Arikan in 2009, is an error correcting code scheme that uses the polarization technique to obtain almost noise-free channels that are suitable for transmission. Polar codes are being widely studied due to their applications on the 5th Generation (5G) of mobile communication systems. In 5G communications, the wireless channel is subject to fading that can significantly degrade the quality of the transmitted signals. In this context, the generalized fading models are useful due to their capacity to model a variety channel conditions ranging from moderate to severe fading by adjusting some parameters. In this work, the authors investigate the performance of polar codes considering the transmission over generalized fading channels. Additionally, the effect of channel correlation is analyzed and a random interleaver is proposed to mitigate the negative impact of the correlation on the system performance. For the system with generalized fading modeled by $\eta-\mu$ distribution and Cyclic Redundancy Check (CRC) Aided Successive Cancellation List (CA-SCL) decoding, the scenario with correlated fading channel and interleaving shows a performance improvement compared to the system without interleaving. In this case, a gain of approximately $3.5 \mathrm{~dB}$ is obtained considering a Bit Error Rate (BER) of $10^{-2}$. Considering the $\alpha-\mu(\alpha=2$ and $\mu=1)$ and the $\kappa-\mu(\kappa=\mu=2)$ fading channels, the uncorrelated systems outperform the correlated ones. Among them, the interleaver improves the performance of the systems. For BER of $10^{-2}$, as an example, the use of fading correlated channel and interleaver shows a performance improvement of $12 \mathrm{~dB}$ and $0.5 \mathrm{~dB}$ for the $\alpha-\mu$ and $\kappa-\mu$ fading channels, respectively.
\end{abstract}

Index Terms-5G, polar codes, wireless communications, generalized fading channels.

\section{INTRODUCTION}

$\mathbf{R}$ ECENT years have seen a fast growing demand of mobile services with greater expectations for Quality of Service (QoS), ultra-low latency and high reliability standards. These requirements motivated the development of the 5 th Generation (5G) of mobile communication systems [1].

There is a variety of challenges that need to be overcome in the context of mobile communications. One of them is the multipath propagation phenomenon [2] in which many replicas of the transmitted signal can simultaneously reach

F. C. P. Barros, F. C. M. Oliveira and M. L. M. G. Alcoforado are with the Post-graduate Program in Systems Engineering, Polytechnic School of Pernambuco, University of Pernambuco, Rua Benfica, 455, Recife, 50.720001, PE, Brazil. E-mails: fcpb@poli.br, fcmo@poli.br and mlmga@poli.br.

W. T. A. Lopes is with the Post-Graduate Program in Electrical Engineering, Department of Electrical Engineering, Federal University of Paraíba, Cidade Universitária, João Pessoa, 58.059-970, PB, Brazil. E-mail: waslon@ieee.org.

The authors would like to express their thanks to the Coordination for the Improvement of Higher Education Personnel (CAPES) - Finance Code 001, PVEX 88881.171292/2018-01 - and the Brazilian National Council for Scientific and Technological Development (CNPq).

Digital Object Identifier: 10.14209/jcis.2022.1 the receiver. When the sum of such replicas is destructive, fading occurs. Another challenge is the presence of noise, such as Additive White Gaussian Noise (AWGN) [3], that can be defined as unwanted disturbance on a given signal. These effects, fading and additive noise, have the potential of significantly changing the transmitted message so that the receiver gets a different message of the one originally sent by the transmitter characterizing an error.

In order to minimize the effects of the channel on the transmitted messages several block-based error correcting codes have been developed, for instance, Reed-Solomon [4], LowDensity Parity-Check [5], [6] and polar codes [7].

This work is focused on polar codes that were introduced by Arikan [8] in 2009. polar codes use the channel polarization technique to obtain almost noise-free channels that are suitable for transmission [9]. These codes, are theoretically significant because they achieve the symmetric capacity for binary discrete memoryless channels (B-DMC). Additionally, they are important in practice due to their relatively low encoding and decoding complexity [8], [10].

Efficient construction methods of polar codes for the binary erasure channels (BECs) and binary symmetric channels (BSCs) were proposed in [11], [12], [13]. The technique called channel polarization [8] works by combining and splitting channels until each combined channel becomes either purely noisy ("bad" bit-channels) or noiseless ("good" bit-channels) when the block length increases. The most suitable channels are chosen for the transmission of information bits, while the inputs to "bad" bit-channels are fixed to zero, known by both transmitter and receiver. These bits are also called frozen bits.

Using Successive Cancellation (SC), polar codes are capable of achieving symmetric capacity with complexity $O=$ $N \log (N)$ on both coding and decoding stages, in which $N$ is the code block length [8].

The main point of the channel polarization technique is the possibility of accessing, in a separate fashion, each coordinate of a $n$-uple of a channel, $W_{N}^{(i)}$, which is generated by making $N$ copies of a channel, $W$, and transmitting information only on suitable coordinates of $W_{N}^{(i)}$ given by the Bhattacharyya Parameter [14]. In order to achieve such transmission it is necessary to define the block length that will be sent through $W_{N}^{(i)}$ and the amount of information bits, $K$, in each block [15].

Initially, the Bhattacharyya parameter was presented for a BEC channel, and gradually extended for other channels. In 2012, Shi et al [16] presented an expression of the Bhattacharyya parameter for Gaussian and Rayleigh fading, including recursive formulas and initial values. They analyzed 
applications concerning the transmission of image and speech. In 2013, Bravo [17] presented two methods for the design of polar codes considering the channel estimation scenario. One for the case of block Rayleigh fading channel with known channel side information and the other for the Rayleigh channel with known channel distribution information. For the second case, the results showed a penalty for the absence of complete information about the channel state.

In 2014, $\mathrm{Si}$ et al [18] proposed, for a fading binary symmetric channel (BSC), a hierarchical scheme based on polar coding for block fading channels. For fading additive exponential noise channels, expansion coding was used in conjunction with polar codes. In 2016, Liu and Ling [19] designed polar codes and polar lattices for independent identically distributed fading channels when the channel state information is not available to the transmitter. For the binary input case, they proposed a design of polar codes through single-stage polarization to achieve the ergodic capacity. In 2018, Fang et al [20] introduced polar codes in a multiple input multiple output free space optical communication system to mitigate turbulence-induced fading. Recently, Niu and Li [21] introduced a systematic framework to analyse and design polar codes under various fast fading channels, including Rayleigh, Rician and Nakagami channels.

The construction of polar codes in this work considers the polar sequence and its corresponding reliability according to the Technical Specification produced by ETSI 3rd Generation Partnership Project (3GPP) [22]. Here, the use of polar codes is extended from the AWGN channel in the direction of generalized fading channels modeled with $\alpha-\mu$ [23], $\kappa-\mu$ [24] and $\eta-\mu$ [25] distributions. Generalized fading models embrace a variety of practical transmission scenarios ranging from moderate to severe fading and include Rayleigh, Rice and Nakagami, as special cases. This paper presents simulations on different fading scenarios with random interleaving [26] and correlation using Cholesky's decomposition method [27]. Here, the main contribution is to analyse the bit error rate (BER) for uncorrelated and correlated systems, with and without interleaving. For the system with generalized fading and CA-SCL decoding, the scenario with correlated fading channel and interleaving shows a performance improvement compared to the system without interleaving.

The remaining of this paper is organized as follows. In Section II, an approach to the polarization technique is presented. Section III deals with polar coding and decoding fundamentals. The generalized fading distributions used in this work is discussed in Section IV. The methodology used to generate correlation in the samples of fading and the interleaving technique used in this work are presented in Section V and Section VI, respectively. Section VII details the system model used in the simulations. The results are presented and discussed in Section VIII while Section IX is devoted to the conclusions.

\section{Channel Polarization}

It is shown in [28] that channel polarization is a technique that generates $N$ independent channels, $W_{N}^{(i)}, 1 \leq i \leq N$, from $N$ copies of a channel $W$. The polarization consists of the fact that a fraction of $W_{N}^{(i)}$ will have mutual information approximately equal to one, and such coordinates are used to send information bits, while the remaining components will have mutual information approximately equal to zero that are not suitable for transmission. The bits transmitted by the later fraction of channels are named frozen bits, known by both transmitter and receiver. The polarization becomes more evident as $N$ gets higher [8]. This technique has two stages: channel combining and channel splitting [14] as described in the next subsections.

\section{A. Channel Combining}

The goal of this stage is to generate a channel $W_{N}: X^{N} \rightarrow$ $Y^{N}$, where $N=2^{n}$ and $n \geq 1$, out of $N$ copies of $W$ [8], where $X$ belongs to the input alphabet and $Y$ belongs to the output alphabet.

When $n=1$, for example, two copies of $W$ are combined in order to produce the vector channel $W_{2}: X^{2} \rightarrow Y^{2}$ with the following transition probabilities

$$
W_{2}\left(y_{1}, y_{2} \mid u_{1}, u_{2}\right)=W\left(y_{1} \mid u_{1} \oplus u_{2}\right) W\left(y_{2} \mid u_{2}\right),
$$

where $W(A \mid B)$ is the conditional probability of $A$ given $B, \oplus$ represents modulo 2 addition, $y_{1}, y_{2}$ are the outputs of the channel $W_{2}$ and $u_{1}, u_{2}$ are the channel inputs [8]. For the channel $W_{2}, x_{1}=u_{1} \oplus u_{2}$ and $x_{2}=u_{2}$.

\section{B. Channel Splitting}

After obtaining $W_{N}$, the following step is to split it into a set of $N$ sub-channels $W_{N}^{(i)}: X \rightarrow Y^{N} \times X^{i-1}, 1 \leq i \leq N$, with the following transition probabilities [8]

$$
W_{N}^{(i)}\left(y_{1}^{N}, u_{1}^{i-1} \mid u_{i}\right)=\sum_{u_{i+1}^{N} \in X^{N-i}} \frac{1}{2^{N-1}} W_{N}\left(y_{1}^{N} \mid u_{1}^{N}\right),
$$

where $\left(y_{1}^{N}, u_{1}^{i-1}\right)$ is the output of the channel $W_{N}^{(i)}$ and $u_{i}$ is the input [8].

\section{POLAR CODING}

The existence of capacity-achieving code sequences was proved by Shannon [29]. However, an example of such sequence was not provided. In 2009, Arikan proposed a class of codes, known as polar codes, that achieve this goal with low encoding and decoding complexities [8].

The main point of polar coding is to access each coordinate of an $n$-tuple of the channel, $W_{N}^{(i)}$, separately and transmit information using the $K$ coordinates with the highest values of mutual information [8].

It is important to define the variables that will be used. Let $N$ be the block length that will be transmitted through a certain memoryless channel, $W_{N}^{(i)}, K$ be the amount of information bits in each block and $R$ be the code rate defined by $R=$ $K / N$ [15]. The information bits are transmitted through the $K$ most reliable coordinates of $W_{N}^{(i)}$ which are chosen according to the Bhattacharyya parameter [8] with initial parameter $z_{0}=$ $e^{-R E_{b} / N_{0}}$ as shown in [30], where $E_{b} / N_{0}$ is a signal-to-noise ratio per bit. 


\section{A. Polar Decoding}

The decoding algorithms used in this paper are called Successive Cancellation (SC) decoding [31] and Cyclic Redundancy Check (CRC) Aided Successive Cancellation List (CA-SCL) decoding [32].

The $\mathrm{SC}$ is able to estimate and decide the message bits based on the structure of the encoder and the channel output [31]. Unlike soft decoding decoding techniques [33], when decoding a bit from a frozen position the decoder automatically assigns a previously known bit, in this work such bit is 0 , to $\hat{u}$, the decoded message sequence. Otherwise, for decoding an information bit, the decoder decision is based on the previous decoded bits and their Log-Likelihood Ratio (LLR) as [8]

$$
L_{N}^{(i)}\left(y_{1}^{(N)}, \hat{u}_{1}^{i-1}\right)=\ln \left(\frac{W_{N}^{(i)}\left(y_{1}^{(N)}, \hat{u}_{1}^{i-1} \mid u_{i}=0\right)}{W_{N}^{(i)}\left(y_{1}^{(N)}, \hat{u}_{1}^{i-1} \mid u_{i}=1\right)}\right),
$$

where $W_{N}^{(i)}$ is the transition probability of a given channel.

When $W$ is an AWGN channel, the transition probability is defined as

$$
W_{N}^{(i)}=\frac{1}{\sigma_{n} \sqrt{2 \pi}} e^{-\frac{\left(y_{i}-x_{i}\right)^{2}}{2 \sigma_{n}^{2}}},
$$

where $\sigma_{n}$ is the standard deviation of the additive Gaussian noise. Then, the initial LLRs can be defined as follows

$$
L_{i}=\frac{2}{\sigma_{n}^{2}} y_{i}
$$

In the SCL decoding algorithm, a set of $L$ parallel SC decoders is used and an ordered list of size $L$ is constantly updated. It is important to mention that the probability of finding the correct sequence increases when the list size $L$ increases. However, there is an increment in the computational complexity [34]. At the end of the decoding process, the correct bit sequence is usually in the final $L$-size list and, in case of fail, it would present smaller likelihood compared to other sequences. In this case, the chosen sequence is the one that has the best likelihood and passes in the CRC test [34], [35].

It is important to mention that, according to the Technical Specification TS 38.212 Version 15.2.0 Release 15 produced by the ETSI 3rd Generation Partnership Project (3GPP) [22], a generator polynomial for the CRC of order $r=11$ should be used for messages with length greater than or equal to 20 for the Uplink Control Information (UCI) encoded by polar codes. In this case, the generator polynomial is given by [22]

$$
g(D)=\left[D^{11}+D^{10}+D^{9}+D^{5}+1\right] .
$$

Considering the addition of a CRC of length $r$ to a polar code $(N, K-r)$, the $K-r$ most reliable bit channels are assigned to the information bits, the next most reliable $r$ channels are assigned to CRC bits and the $N-K$ remaining bit channels are frozen. By this way, the overall system code rate is $(K / N)$ [35].

\section{Generalized FADING}

In wireless communications, fading is a variation of the attenuation of a signal as a result of the changes of various channel parameters. One effect of fading is the loss in average path power that the signal suffers over distances [36].

The well-known fading distributions, such as Rayleigh, Nakagami- $m$, Hoyt and Rice, were derived based on the assumption of a homogeneous transmission medium [37]. The $\alpha-\mu, \kappa-\mu$ and $\eta-\mu$ fading distributions are proposed to properly contemplate non-homogeneous channels [37], [38], [39].

\section{A. $\alpha-\mu$ fading}

The $\alpha-\mu$ fading distribution is a rewritten form of generalized Gamma distribution and explores the non linearity of the propagation medium by using a power parameter $\alpha$. In this distribution, $\mu$ represents the number of multipath propagation clusters used in the system [40]. The fading envelope, $V$, is defined as follows

$$
V^{\alpha}=\sum_{i=1}^{\mu}\left(F_{i}^{2}+G_{i}^{2}\right)
$$

where $F_{i}$ and $G_{i}$ are mutually independent Gaussian processes, with $E\left[F_{i}\right]=E\left[G_{i}\right]=0$ and $E\left[F_{i}^{2}\right]=E\left[G_{i}^{2}\right]=$ $\hat{v}^{\alpha} / 2 \mu, \hat{v}=\sqrt[\alpha]{E\left[V^{\alpha}\right]}[41]$ and $E[\cdot]$ is the expected value operator. The probability distribution function is given by

$$
f_{V}(v)=\frac{\alpha \mu^{\mu} v^{\alpha \mu-1}}{\hat{v}^{\alpha \mu} \Gamma(\mu)} \exp \left(-\mu \frac{v^{\alpha}}{\hat{v}^{\alpha}}\right),
$$

in which $\alpha>0$ can be arbitrarily chosen, $\mu>0, v \geq 0$, and $\Gamma(\cdot)$ is the Gamma function [41].

The $\alpha-\mu$ fading distribution includes Nakagami- $m$, Negative Exponential, Weibull, Semi-Gaussian and Rayleigh distributions as special cases depending on the suitable choice of the parameters $\alpha$ and $\mu$. For instance, when $\alpha=2$ and $\mu=1$, the $\alpha-\mu$ distribution becomes the Rayleigh distribution [41].

\section{B. $\kappa-\mu$ fading}

The $\kappa-\mu$ fading distribution is used to describe the smallscale variation of a certain fading signal in a line-of-sight condition. The signal is composed of $\mu$ clusters, each one with a dominant component, that propagates on a non-homogeneous medium. The $\kappa-\mu$ fading distribution also considers the independence of the in-phase and quadrature components [42]. The envelope, $V$, for the $\kappa$ - $\mu$ fading distribution is given by

$$
V^{2}=\sum_{i=1}^{\mu}\left(F_{i}+p_{i}\right)^{2}+\sum_{i=1}^{\mu}\left(G_{i}+q_{i}\right)^{2},
$$

where $F_{i}$ and $G_{i}$ are mutually independent Gaussian processes, with $E\left[F_{i}\right]=E\left[G_{i}\right]=0$ and $E\left[F_{i}^{2}\right]=E\left[G_{i}^{2}\right]=\sigma^{2}$, $p_{i}$ and $q_{i}$ are the mean values of the in-phase and quadrature components, respectively, of the multipath waves of cluster $i$ [42]. The probability density function is given by

$$
\begin{array}{r}
f_{V}(v)=\frac{2 \mu(1+k)^{\frac{\mu+1}{2}}}{k^{\frac{\mu-1}{2}} \exp (\mu \kappa)} \frac{v^{\mu}}{\hat{v}^{\mu+1}} \exp \left(-\mu \frac{(1+\kappa) v^{2}}{\hat{v}^{2}}\right) \\
I_{\mu-1}\left(\frac{2 \mu \sqrt{\kappa(1+\kappa)} v}{\hat{v}}\right),
\end{array}
$$

in which $\hat{v}=\sqrt{E\left[V^{2}\right]}$ and $I_{m}(\cdot)$ is the modified Bessel function of first species and arbitrary order $m$ [42]. 
The $\kappa-\mu$ fading distribution includes Nakagami- $m$, Rice, Semi-Gaussian and Rayleigh distributions as special cases depending on the suitable choice of the parameters $\kappa$ and $\mu$. For instance, when $\mu=1$, the $\kappa-\mu$ distribution becomes the Rice distribution and, when $\kappa \rightarrow 0$, it becomes the Nakagami$m$ distribution [37].

\section{C. $\eta$ - $\mu$ fading}

The $\eta-\mu$ fading distribution is used to describe the smallscale variation of a given fading channel without direct line-ofsight to the receiver. This fading distribution has two formats but this work is focused on Format I. In this case, the inphase and quadrature components are independent, $\eta$ is the ratio between the in-phase and quadrature power and $\mu$ is the number of clusters [42]. Then the fading envelope, $V$, is defined as

$$
V^{2}=\sum_{i=1}^{\mu}\left(F_{i}^{2}+G_{i}^{2}\right)
$$

where $F_{i}$ and $G_{i}$ are mutually independent Gaussian processes, with $E\left[F_{i}\right]=E\left[G_{i}\right]=0$ and $E\left[F_{i}^{2}\right]=\sigma_{F}^{2}$, $E\left[G_{i}^{2}\right]=\sigma_{G}^{2}$, and $\hat{v}=\sqrt{E\left[V^{2}\right]}[42]$. The probability density function is given by

$$
\begin{array}{r}
f_{V}(v)=\frac{4 \sqrt{\pi} \mu^{\mu+\frac{1}{2}} h^{\mu} v^{2 \mu}}{\Gamma(\mu) H^{\mu-\frac{1}{2}} \hat{v}^{2 \mu+1}} \exp \left(-\frac{2 \mu h v^{2}}{\hat{v}^{2}}\right) \\
I_{\mu-1}\left(\frac{2 \mu H v^{2}}{\hat{v}^{2}}\right),
\end{array}
$$

in which $h=\left(2+\eta+\eta^{-1}\right) / 4$ and $H=\left(\eta^{-1}-\eta\right) / 4$.

The $\eta-\mu$ fading distribution includes Nakagami- $m$, Hoyt, one-sided Gaussian and Rayleigh distributions as special cases depending on the suitable choice of the parameters $\eta$ and $\mu$. For instance, when $\mu=1$, the $\eta-\mu$ distribution becomes the Hoyt distribution and, when $\eta \rightarrow 1$ and $\mu=1$, it becomes the Rayleigh distribution [37].

\section{Correlated Fading Channels}

In wireless communication systems the most frequent scenario is the absence of line-of-sight path between the transmitter and the receiver. Thus, there is a non-line-of-sight multipath propagation scenario. It is shown in [43] that the received replicas of the signal are often correlated and, as a consequence, the quality of transmission is lowered.

In this work, the introduction of correlation in the fading model is performed by using Cholesky decomposition [44] followed by the inverse discrete Fourier transform [45]. Considering a correlation coefficient $\lambda$, the correlated fading coefficients are obtained using a $2 \times 2$ correlation matrix, $M$, defined as

$$
M=\left[\begin{array}{cc}
E\left[F_{i}^{2}\right] & \lambda \sqrt{E\left[F_{i}^{2}\right] \cdot E\left[G_{i}^{2}\right]} \\
\lambda \sqrt{E\left[F_{i}^{2}\right] \cdot E\left[G_{i}^{2}\right]} & E\left[G_{i}^{2}\right]
\end{array}\right],
$$

The matrix in Equation (13) is decomposed by using Cholesky approach in order to generate a matrix $T$ of the same order of $M$.

The next step is the generation of uncorrelated signal by using Gaussian distributions for the in-phase, $P h_{i}$, and quadrature, $Q_{i}$, components. Then, they are both shaped by a Doppler filter [45], $\mathbb{H}(\cdot)$, followed by the inverse discrete Fourier transform. The Doppler filter with $N$ samples is defined as

$$
\mathbb{H}(d)=\left\{\begin{array}{l}
0, \text { if } d=0 ; \\
\sqrt{\frac{1}{\left.2 \sqrt{1-\left(\frac{d}{N f_{m}}\right.}\right)}}, \text { if } d=1,2, \ldots, d_{m}-1 ; \\
\sqrt{\frac{d_{m}}{2}\left[\frac{\pi}{2}-\tan ^{-1}\left(\frac{d_{m}-1}{\sqrt{2 d_{m}-1}}\right)\right]}, \text { if } d=d_{m} ; \\
0, \text { if } d=d_{m}+1, \ldots, N-d_{m}-1 ; \\
\sqrt{\frac{d_{m}}{2}\left[\frac{\pi}{2}-\tan ^{-1}\left(\frac{d_{m}-1}{\sqrt{2 d_{m}-1}}\right)\right]}, \text { if } d=N-d_{m} ; \\
\sqrt{\left.\frac{1}{2 \sqrt{1-\left(\frac{d}{N f_{m}}\right.}}\right)}, \text { if } d=N-d_{m}+1, \ldots, N-1 ;
\end{array}\right.
$$

in which $d_{m}=\left\lfloor f_{m} N\right\rfloor$ and $f_{m}$ is the maximum frequency deviation (Doppler frequency) [45].

The final step to generate the correlated components is

$$
\left[P h_{i(\mathrm{corr})} \quad Q_{i(\mathrm{corr})}\right]=\left[\begin{array}{ll}
P h_{i} & Q_{i}
\end{array}\right] \cdot T,
$$

where $P h_{i}$ and $Q_{i}$ are, respectively, the in-phase and the quadrature uncorrelated Gaussian samples for cluster $i$, where $1 \leq i \leq \mu$.

It is worth to mention that the new components $P h_{i(\text { corr })}$ and $Q_{i \text { (corr) }}$ are correlated Gaussian distributions for in-phase and quadrature components for cluster $i$.

Figs. 1, 2 and 3 depict the $\kappa-\mu$ fading coefficients for $f_{m}=$ $1.5,5$ and $15 \mathrm{~Hz}$, respectively. The coefficients were generated using a sampling frequency of 600 kbauds, $\kappa=2, \mu=2$ and $N=1024$. As expected, faster variations of the channel coefficients are obtained by increasing the value of $f_{m}$.

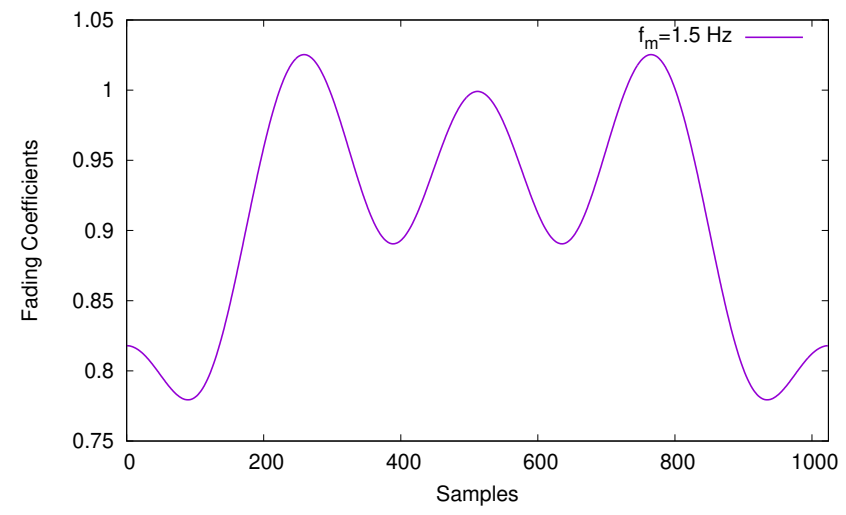

Fig. 1. Fading coefficients for $\kappa-\mu$ fading considering $f_{m}=1.5 \mathrm{~Hz}, \kappa=2$, $\mu=2$ and $N=1024$

\section{INTERLEAVING}

A interleaver is a device that takes a sequence of input symbols from a given alphabet and produces another sequence composed by the same input symbols in a different order. In this work the application of the interleaving is to reduce the correlation among information bits in order to prevent burst errors [46], [47]. 


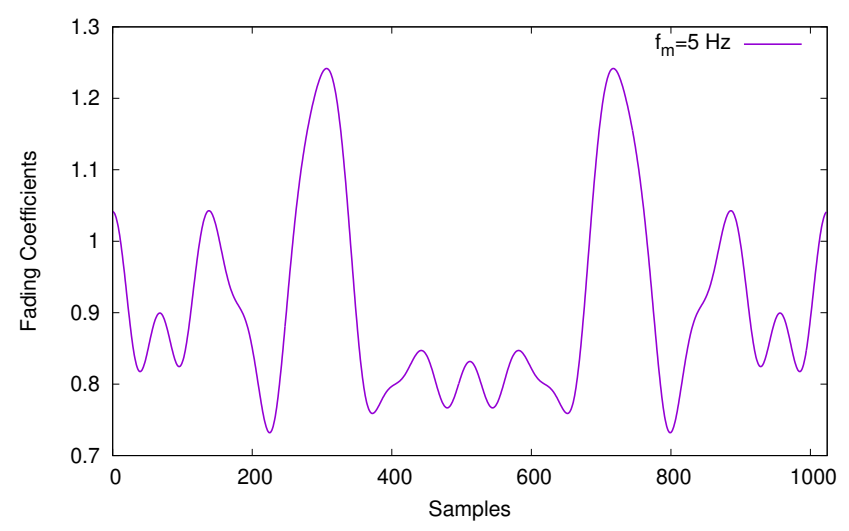

Fig. 2. Fading coefficients for $\kappa-\mu$ fading considering $f_{m}=5 \mathrm{~Hz}, \kappa=2$, $\mu=2$ and $N=1024$.

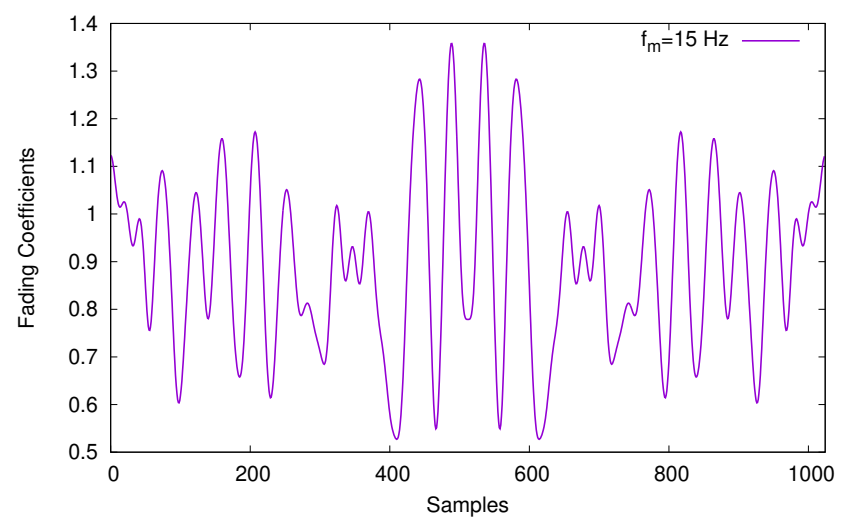

Fig. 3. Fading coefficients for $\kappa$ - $\mu$ fading considering $f_{m}=15 \mathrm{~Hz}, \kappa=2$, $\mu=2$ and $N=1024$.

In Figs. 1, 2 and 3, there are some time intervals in which a sequence of fading coefficients presents small gain. In this case, the sequence of transmitted symbols will be severely attenuated leading burst errors.

It is important to point that if one decides to use an interleaver, it is also necessary to use a deinterleaver in order to return the symbols to the original order. Only after deinterleaving, the decoding process can take place.

Interleavers can be divided in two types: block and convolutional interleavers [48]. In a block interleaver the data are commonly structured along the rows of a permutation matrix and read out along the columns [48]. A convolutional interleaver is based on shift registers in order to multiplex the data [49]. This work uses a block random interleaver and it is generated from a random permutation based on a random source.

\section{System Model}

The simulated system is an extended version of the system found in [50] and transmits information with using polar coding and BPSK modulation through an AWGN channel with generalized correlated fading [51]. The random interleaver [26] used in this work has the same length $N$ of the polar code. Therefore, the received signal $r(t)$, is given by

$$
r(t)=\alpha(t) s(t)+n(t),
$$

in which $\alpha(t)$ is the fading envelope, $s(t)$ is the transmitted signal and $n(t)$ is the additive Gaussian noise.

Fig. 4 depicts a basic version of the system without the interleaver while Fig. 5 shows the block diagram of the system with a decoder followed by an interleaver, at the transmitter side, and the deinterleaver just before the decoder, at the receiver side. In both figures, when the Encoder block represents a polar encoding, the Decoder block is an SC decoder. Otherwise, when the Encoder block is composed by a polar encoder, as an inner encoder, concatenated with a CRC encoder, as an outer encoder, the Decoder block is a CA-SCL decoder [32].

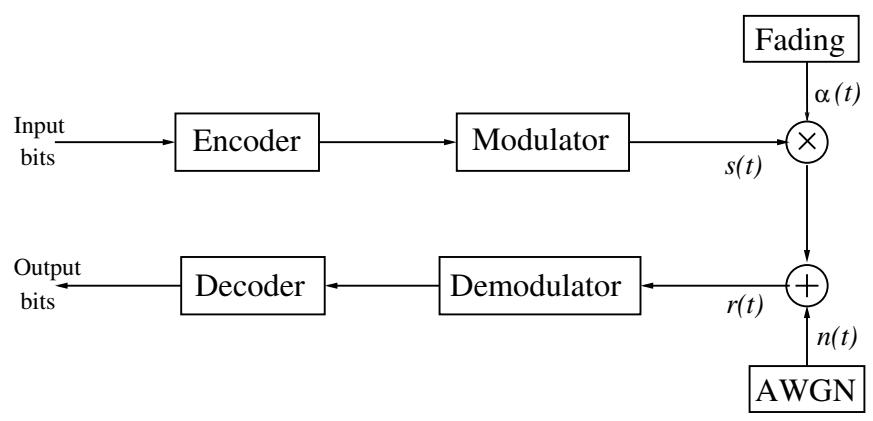

Fig. 4. Block diagram of the proposed system without interleaving.

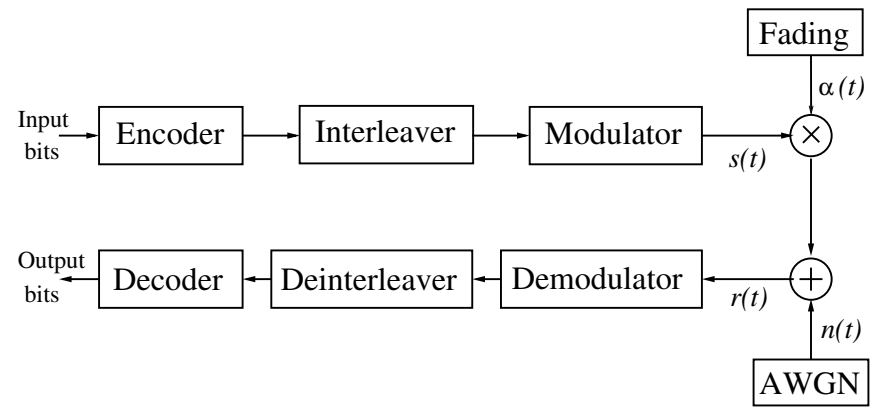

Fig. 5. Block diagram of the proposed system with interleaving.

\section{RESULTS}

The systems depicted in Figs. 4 and 5 were implemented in Matlab ${ }^{\circledR}$ using Monte Carlo method with a minimum of 20,000 simulations per point. The Doppler frequency, $f_{m}$, used in all simulations presented in this work was $1 \mathrm{~Hz}$. The metric used to evaluate the system performance is the bit error rate (BER) as a function of the signal-to-noise ratio $\left(E_{b} / N_{0}\right)$, in dB. All results presented also consider $K=512$ information bits in a block length of $N=1024$ bits, correlation coefficient $\lambda=0.8$ for the correlated channels and the interleaver length is 1024 bits. In the simulations, the criteria used to adjust the parameters $\alpha, \eta, \kappa$ and $\mu$ of the generalized fading models is twofold. First, the parameters correspond to special cases of fading, as Rayleigh, Rice and Nakagami. Second, the 
parameters embrace practical transmission scenarios ranging from moderate to severe fading.

Considering the $\alpha-\mu$ fading and the SC decoding, it is possible to see in Fig. 6, for $\alpha=2$ and $\mu=1$, that the system has better performance when the fading channel is uncorrelated. For correlated fading channels, the use of the interleaver enhances the system performance from the correlated fading case in the direction of the uncorrelated case. This happens because burst transmission errors can degrade the system performance and interleaving shuffles the transmitted sequences improving the performance in terms of error detection and correction. For BER of $10^{-2}$, for example, the scenario using the uncorrelated channel shows a significant performance improvement when compared to the cases of correlated fading channels with or without interleaver, where the $E_{b} / N_{0}$ gains are approximately of $7 \mathrm{~dB}$ and $12 \mathrm{~dB}$, respectively. It is worth to remember that, in the case of $\alpha=2$ and $\mu=1$, the $\alpha-\mu$ distribution becomes the Rayleigh distribution.

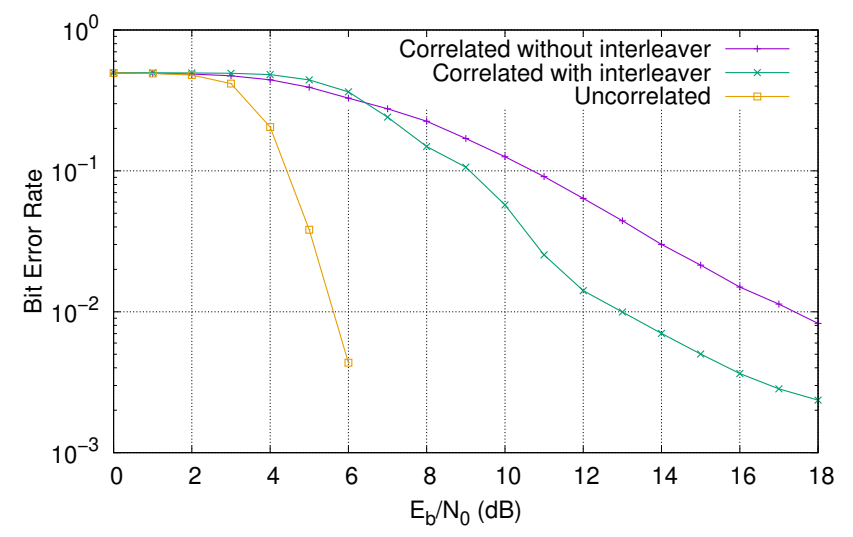

Fig. 6. Comparison of different scenarios of $\alpha-\mu$ fading considering $N=$ 1024 bits, $\lambda=0.8, \alpha=2, \mu=1$ and SC decoding.

In Fig. 7, for $\alpha-\mu$ fading channel with $\alpha=2$ and $\mu=1$, 2 and 4, all curves correspond to a system with correlation, interleaving and SC decoding. It can be observed that as $\mu$ ranges from 1 to 4 the system performance is improved. This is expected because the higher is $\mu$, the lower is the fading intensity and the corresponding BER. That happens because an increase in the number of clusters leads to a higher probability of receiving a sample of the signal than can be correctly decoded. Considering the BER of $10^{-2}$, for example, the scenario with $\mu=4$ shows a significant performance improvement compared to the case with $\mu=1$, with a corresponding $E_{b} / N_{0}$ gain of approximately $3.5 \mathrm{~dB}$.

In Fig. 8, scenarios for $\alpha=2$ and $\mu=1,2$ and 4 , without the interleaver are presented. It can be noted the same behaviour found in Fig. 7 where as $\mu$ increases the system performs better. Considering, for instance, the curves generated using SC decoding, for BER equals to $10^{-2}$, the case where $\mu=4$ shows a significant performance improvement when compared to $\mu=2$ and $\mu=1$, presenting gains of 1 $\mathrm{dB}$ and $3 \mathrm{~dB}$, respectively. Considering the curves generated using CA-SCL decoding, for the same BER, the gains are

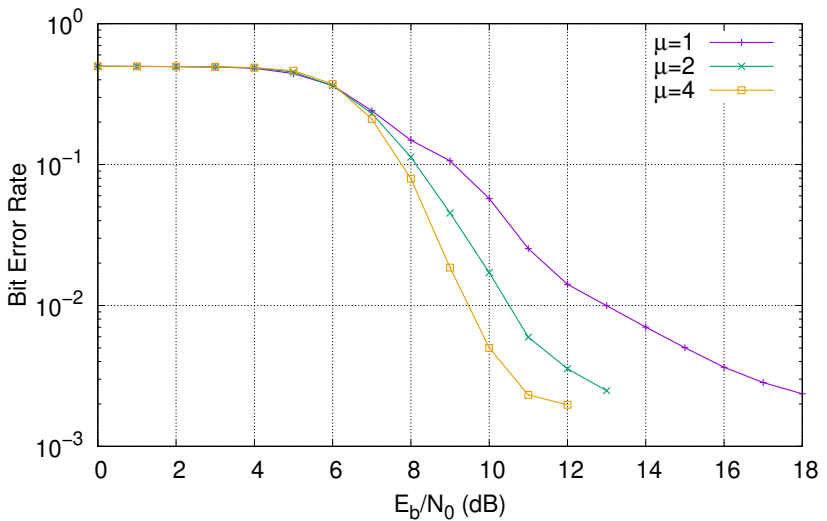

Fig. 7. Comparison of different scenarios of $\alpha-\mu$ fading with interleaving depth of $N=1024$ bits, correlation coefficient $\lambda=0.8$ and SC decoding. In this figure $\alpha=2$ and $\mu=1,2$ and 4 .

approximately $0.5 \mathrm{~dB}$ and $2 \mathrm{~dB}$, for the cases with $\mu=2$ and $\mu=1$, respectively. It is important to mention that for $\mu=4$, the use of CA-SCL algorithm shows a performance improvement, with a gain of $2 \mathrm{~dB}$, when compared to the case using SC decoding for the BER of $10^{-2}$.

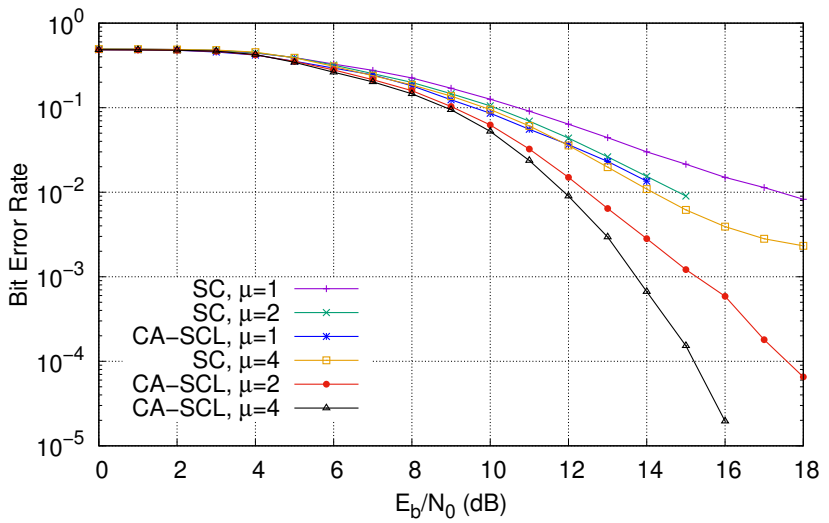

Fig. 8. Comparison of different scenarios of $\alpha-\mu$ fading for $N=1024$ bits, correlation coefficient $\lambda=0.8$ and SC/CA-SCL decoding. In this figure $\alpha=2$ and $\mu=1,2$ and 4 .

Now, consider the second type of generalized fading modeled by $\eta-\mu$ distribution. It can be seen in Fig. 9, for $\eta=1$ and $\mu=0.5$ which corresponds to the Nakagami- $m$ fading with $m=1$, that the uncorrelated system has superior performance when compared to the correlated ones. Among the correlated ones the interleaver improves the performance of the system. For example, the curves generated in systems with the SC decoder, for the BER of $10^{-2}$, the scenario using correlated channel with interleaver shows a performance improvement gain of $4 \mathrm{~dB}$ when compared to the case without interleaver. For the systems with CA-SCL and the same BER, this gain is approximately $3 \mathrm{~dB}$. For a fixed $\mu$ value, the use of CASCL decoder improves the performance of the system when compared with the system with SC decoder.

Finally, considering $\kappa-\mu$ fading channel, it is possible to note in Fig. 10, where $\kappa=\mu=2$, that the uncorrelated system 


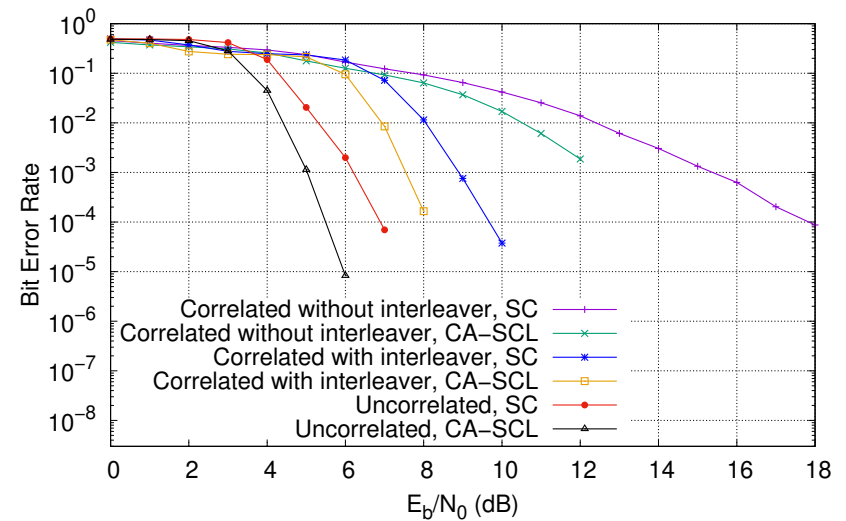

Fig. 9. Comparison of different scenarios of $\eta-\mu$ fading for $N=1024$ bits, $\lambda=0.8, \eta=1, \mu=0.5$ and SC/CA-SCL decoding.

has superior performance when compared to the correlated ones. Among the correlated ones the interleaver improves the performance of the system and such behaviour is also seen in Figs. 8 and 9. For the curves generated in systems with the SC decoding and BER of $10^{-2}$, the use of fading correlated channel and interleaver shows a performance improvement compared to the case without interleaver with gain of $0.5 \mathrm{~dB}$. For the curves generated in systems with CA-SCL decoding at the same BER, this gain is also approximately $0.5 \mathrm{~dB}$. As the expected, the CA-SCL scenario achieves better performance than the SC scenario.

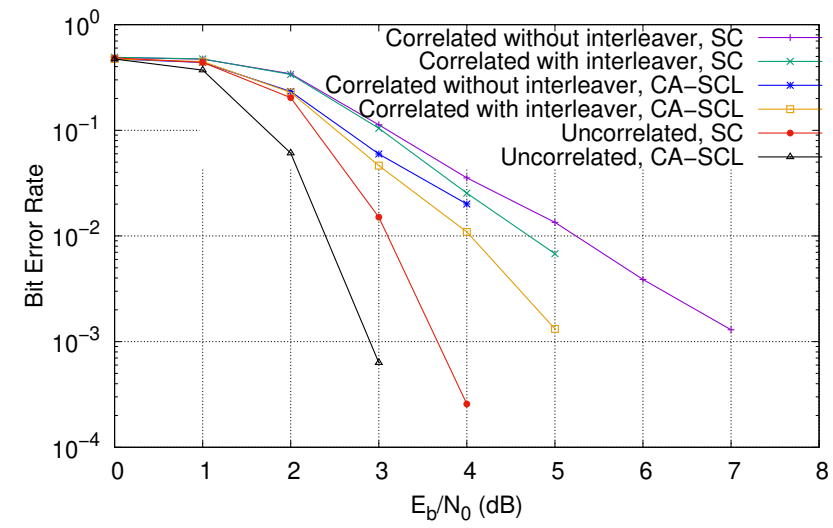

Fig. 10. Comparison of different scenarios of $\kappa-\mu$ fading for $N=1024$ bits, $\lambda=0.8, \kappa=2, \mu=2$ and SC/CA-SCL decoding.

\section{CONClusions And Future Works}

This paper presented a performance analysis of polar codes in the context of generalized fading channels considering $\eta$ $\mu, \alpha-\mu$ and $\kappa-\mu$ fading distributions. An important aspect of those distributions is the fact that they can provide an unified analysis of the influence of different types of fading in the performance of the communications system, such as Hoyt, Rice, Nakagami- $m$, Rayleigh, and one-sided Gaussian. Additionally, the effects of the correlation of the fading samples were discussed and a random interleaver was proposed to enhance the performance of the overall system. Finally, the use of CA-SCL algorithm improves the performance results, when compared with the SC decoding considering all scenarios presented.

For future works, different interleaving algorithms [52] can be used as well as other decoding schemes such as Belief Propagation [53], other modulation techniques [54] and transmission by multiple antennas [55].

\section{REFERENCES}

[1] Z. B. Kaykac Egilmez, L. Xiang, R. G. Maunder, and L. Hanzo, "The development, operation and performance of the 5G polar codes," IEEE Commun. Surveys Tuts., vol. 22, no. 1, pp. 96-122, 2020. doi: 10.1109/COMST.2019.2960746.

[2] W. T. A. Lopes, Y. Xue, and E. S. Sousa, "Performance of modulation diversity with polar encoding in Rayleigh fading channel," in Proceedings of the 16th ACM Int. Symp. Performance Evaluation of Wireless Ad Hoc, Sensor, and Ubiquitous Networks (PE-WASUN'2019), Miami, FL, USA, November 2019, pp. 51-55. doi: 10.1145/3345860.3361514.

[3] M. El-Khamy, H. Mahdavifar, G. Feygin, J. Lee, and I. Kang, "Relaxed polar codes," IEEE Trans. Inf. Theory, vol. 63, no. 4, pp. 1986-2000, April 2017. doi: 10.1109/TIT.2017.2657691.

[4] W. Li, Z. Wang, and H. Jafarkhani, "Repairing Reed-Solomon codes over $G F\left(2^{\ell}\right)$," IEEE Commun. Lett., vol. 24, no. 1, pp. 34-37, 2020. doi: 10.1109/LCOMM.2019.2950922.

[5] Z. Wu and W. Kang, "A time sharing hybrid probabilistic shaping scheme for nonbinary LDPC codes," IEEE Access, vol. 8, pp. 65488 65 497, 2020. doi: 10.1109/ACCESS.2020.2985864.

[6] A. L. Scharf, B. F. Uchôa-Filho, B. F. da Silva, and D. L. Ruyet, "Low-complexity tree-based iterative decoding for coded SCMA," Journal of Commun. Inf. Systs., vol. 35, no. 1, pp. 180-188, 2020. doi: $10.14209 /$ jcis.2020.19.

[7] M. Chiu, "Interleaved polar (i-polar) codes," IEEE Trans. Inf. Theory, vol. 66, no. 4, pp. 2430-2442, 2020. doi: 10.1109/TIT.2020.2969155.

[8] E. Arikan, "Channel polarization: A method for constructing capacityachieving codes for symmetric binary-input memoryless channels," IEEE Trans. Inf. Theory, vol. 55, no. 7, pp. 3051-3073, July 2009. doi: 10.1109/TIT.2009.2021379.

[9] C. Condo, V. Bioglio, H. Hafermann, and I. Land, "Practical product code construction of polar codes," IEEE Trans. Signal Process., vol. 68, pp. 2004-2014, 2020. doi: 10.1109/TSP.2020.2981766.

[10] E. Arikan and E. Telatar, "On the rate of channel polarization," in Proceedings of the 2009 IEEE Int. Symp. Inf. Theory, ser. ISIT'09, vol. 3. Coex, Seoul, Korea: IEEE Press, 2009. ISBN 9781424443123 pp. 1493-1495. doi: 10.1109/ISIT.2009.5205856.

[11] I. Tal and A. Vardy, "How to construct polar codes," IEEE Trans. Inf. Theory, vol. 59, no. 10, pp. 6562-6582, 2013. doi: 10.1109/TIT.2013.2272694.

[12] R. Mori and T. Tanaka, "Performance of polar codes with the construction using density evolution," IEEE Commun. Lett., vol. 13, no. 7, pp. 519-521, 2009. doi: 10.1109/LCOMM.2009.090428.

[13] R. Pedarsani, S. H. Hassani, I. Tal, and E. Telatar, "On the construction of polar codes," in 2011 IEEE Int. Symp. Inf. Theory Proceedings. IEEE, 2011, pp. 11-15. doi: 10.1109/ISIT.2011.6033724.

[14] Z. Babar, Z. B. Kaykac Egilmez, L. Xiang, D. Chandra, R. G. Maunder, S. X. Ng, and L. Hanzo, "Polar codes and their quantum-domain counterparts," IEEE Commun. Surveys Tuts., vol. 22, no. 1, pp. 123 155, 2020. doi: 10.1109/COMST.2019.2937923.

[15] J. Li, Z. Gao, and Y. Lv, "Gaussian approximation optimized SC- flip decoding algorithm of polar codes," in 2020 IEEE 4th Information Technology, Networking, Electronic and Automation Control Conf. (ITNEC), vol. 1, 2020, pp. 1124-1127. doi: 10.1109/ITNEC48623.2020.9084888.

[16] P. Shi, W. Tang, S. Zhao, and B. Wang, "Performance of polar codes on wireless communication channels," in The IEEE 14th Int. Conf. Commun. Technol.. IEEE, 2012, pp. 1134-1138. doi: 10.1109/ICCT.2012.6511367.

[17] A. Bravo-Santos, "Polar codes for the Rayleigh fading channel," IEEE Commun. Lett., vol. 17, no. 12, pp. 2352-2355, 2013. doi: 10.1109/LCOMM.2013.111113.132103.

[18] H. Si, O. O. Koyluoglu, and S. Vishwanath, "Polar coding for fading channels: Binary and exponential channel cases," IEEE Trans. Commun., vol. 62, no. 8, pp. 2638-2650, 2014. doi: 10.1109/TCOMM.2014.2345399. 
[19] L. Liu and C. Ling, "Polar codes and polar lattices for independent fading channels," IEEE Trans. Commun., vol. 64, no. 12, pp. 49234935, 2016. doi: 10.1109/TCOMM.2016.2613109.

[20] J. Fang, M. Bi, S. Xiao, G. Yang, L. Liu, Y. Zhang, and W. Hu, "Polar-coded MIMO FSO communication system over Gamma-Gamma turbulence channel with spatially correlated fading," Journal of Opt. Commun. Netw., vol. 10, no. 11, pp. 915-923, 2018. doi: 10.1364/JOCN.10.000915.

[21] K. Niu and Y. Li, "Polar codes for fast fading channel: Design based on polar spectrum," IEEE Trans. Veh. Technol., vol. 69, no. 9, pp. $10103-$ 10114, 2020. doi: 10.1109/TVT.2020.3005914.

[22] 3GPP, "5G; NR; Multiplexing and channel coding (3GPP TS 38.212 version 15.2.0 Release 15)," ETSI, TS 38.212, 07 2018, version 15.2.0. [Online]. Available: https://www.etsi.org/deliver/etsi_ts/138200 138299/138212/15.02.00_60/ts_138212v150200p.pdf

[23] A. Khatoon and M. Sharique, "Performance of GSM and GSM-SM over $\alpha-\mu$ fading channel model," in TENCON 2019 - IEEE Region 10 Conf. (TENCON), 2019, pp. 1981-1985. doi: 10.1109/TENCON.2019.8929377.

[24] R. Singh and M. Rawat, "Outage analysis of double shadowed $\kappa-\mu$ fading channels," in 2019 10th Int. Conf. Computing, Commun. Netwo. Technol. (ICCCNT), 2019, pp. 1-4. doi: 10.1109/ICCCNT45670.2019.8944440.

[25] C. Priyanka and V. Nithya, "Physical scenario perusal of eta-mu fading channels," in 2015 Int. Conf. Commun. Signal Process. (ICCSP), 2015, pp. 0708-0712. doi: 10.1109/ICCSP.2015.7322582.

[26] M. Bilim, N. Kapucu, and I. Develi, "A new approach to random interleavers for traditional IDMA systems," in 2012 IEEE Symp. on Comput. Commun. (ISCC), 2012, pp. 000 209-000 212. doi: 10.1109/ISCC.2012.6249295.

[27] H. Leng, G. Fu, M. Jiang, and L. Zhong, "Multivariate correlation degradation parameters life assessment method of component based on the Cholesky factorization," in 2018 Prognostics and System Health Management Conf. (PHM-Chongqing), 2018, pp. 462-467. doi: 10.1109/PHM-Chongqing.2018.00085.

[28] R. M. Oliveira and R. C. de Lamare, "Non-uniform channel polarization and design of rate-compatible polar codes," in 2019 16th Int. Symp. Wireless Commun. Syst. (ISWCS), 2019, pp. 537-541. doi: 10.1109/ISWCS.2019.8877311.

[29] C. E. Shannon, "A mathematical theory of communication," The Bell System Technical Journal, vol. 27, no. 3, pp. 379-423, 7 1948. doi: 10.1002/j.1538-7305.1948.tb01338.x.

[30] H. Vangala, E. Viterbo, and Y. Hong, "A comparative study of polar code constructions for the AWGN channel," CoRR, vol. abs/1501.02473, 2015. [Online]. Available: http://arxiv.org/abs/1501.02473

[31] K. M. Prakash and G. S. Sunitha, "Efficient high performance successive cancelation decoder for polar code," in 2018 3rd IEEE Int. Conf. Recent Trends in Electronics, Information Communication Technology (RTEICT), 2018, pp. 929-933. doi: 10.1109/RTEICT42901.2018.9012094.

[32] K. Niu and K. Chen, "CRC-aided decoding of polar codes," IEEE Commun. Lett., vol. 16, no. 10, pp. 1668-1671, October 2012. doi: 10.1109/LCOMM.2012.090312.121501.

[33] M. L. M. G. Alcoforado, L. Bulhões, A. V. Andrade, F. Madeiro, and V. C. da Rocha Jr., "Data transmission using convolutional codes and bidirectional soft output Viterbi algorithm over the two-user binary adder channel," Journal Commun. Inf. Syst., vol. 32, no. 1, pp. 126-132, 2017. doi: $0.14209 /$ jcis.2017.13

[34] I. Tal and A. Vardy, "List decoding of polar codes," IEEE Trans. Inf. Theory, vol. 61, no. 5, pp. 2213-2226, 2015. doi: 10.1109/ISIT.2011.6033904.

[35] T. Murata and H. Ochiai, "On design of CRC codes for polar codes with successive cancellation list decoding," in 2017 IEEE Int. Symp. Inf. Theory (ISIT). IEEE, 2017, pp. 1868-1872. doi: 10.1109/ISIT.2017.8006853.

[36] A. Goldsmith, Wireless Communications. USA: Cambridge University Press, 2005. ISBN 978-05-2183-716-3.

[37] G. Fraidenraich and M. D. Yacoub, "The $\alpha-\eta-\mu$ and $\alpha-\kappa-\mu$ fading distributions," in 2006 IEEE Ninth Int. Symp. on Spread Spectrum Techniques and Applications, 2006, pp. 16-20. doi: 10.1109/ISSSTA.2006.311725.

[38] F. R. A. Parente, F. du Pin Calmon, and J. C. S. Santos Filho, "Asymptotic system performance over generalized fading channels with application to maximal-ratio combining," Journal Commun. Inf. Syst., vol. 35, no. 1, pp. 171-180, 2020. doi: $10.14209 /$ jcis.2020.18.

[39] V. M. Rennó, R. A. A. de Souza, and M. D. Yacoub, "Analysis of energy detection with noise uncertainty over $\alpha-\eta-\kappa-\mu$ fading channel," Journal Commun. Inf. Systems, vol. 34, no. 1, pp. 171-180, 2019. doi: 10.14209/jcis.2019.18
[40] N. Vasić, D. Stefanović, D. Rancić, D. Milić, and D. Dosić, "Statistics of simultaneous Rice, Nakagami- $m$ and $\alpha-\mu$ fadings after SC combining," in 2020 19th Int. Symp. Infotech-Jahorina (INFOTEH), 2020, pp. 1-4. doi: 10.1109/INFOTEH48170.2020.9066301.

[41] M. D. Yacoub, "The $\alpha-\mu$ distribution: A physical fading model for the Stacy distribution," IEEE Trans. Veh. Technol., vol. 56, no. 1, pp. 27-34, 2007. doi: $10.1109 /$ TVT.2006.883753.

[42] C. R. N. da Silva, E. J. Leonardo, and M. D. Yacoub, "Product of two envelopes taken from $\alpha-\mu, \kappa-\mu$, and $\eta-\mu$ distributions," IEEE Trans. Commun., vol. 66, no. 3, pp. 1284-1295, 2018. doi: 10.1109/TCOMM.2017.2773510.

[43] S. Loyka and A. Kouki, "The impact of correlation on multi-antenna system performance: correlation matrix approach," in IEEE 54th Vehicular Tech. Conf. (VTC Fall 2001). Proceedings (Cat. No.01CH37211), vol. 2, 2001, pp. 533-537. doi: 10.1109/VTC.2001.956826.

[44] Keli Zhang, Zhefeng Song, and Yong Liang Guan, "Cholesky decomposition model for correlated MRC diversity systems in Nakagami fading channels," in Proceedings IEEE 56th Vehicular Tech. Conf., vol. 3, 2002, pp. 1515-1519. doi: 10.1109/VETECF.2002.1040469.

[45] D. J. Young and N. C. Beaulieu, "The generation of correlated Rayleigh random variates by inverse discrete Fourier transform," IEEE Transa. Commun., vol. 48, no. 7, pp. 1114-1127, 2000. doi: 10.1109/26.855519.

[46] S. Lin, S. Lin, and Y. Li, "An enhanced method for turbo code interleavers," in 2019 Int. Symp. on Intelligent Signal Processing and Communication Systems (ISPACS), 2019, pp. 1-2. doi: 10.1109/ISPACS48206.2019.8986335.

[47] R. M. Duarte, M. S. Alencar, W. T. A. Lopes, F. B. S. Carvalho, W. J. L. Queiroz, and D. B. T. Almeida, "Performance of cell-free systems with channel reciprocity errors," Radioengineering, vol. 30, no. 1, pp. 237 249, April 2021. doi: 10.13164/re.2021.0237.

[48] Y. Gagiev, A. Trushanin, R. Maslennikov, A. Lomayev, and A. Kostin, "Block interleaver design for the single carrier modulation in IEEE 802.11ay," in 2018 24th Asia-Pacific Conf. on Communications (APCC), 2018, pp. 57-61. doi: 10.1109/APCC.2018.8633499.

[49] Y. Xu, Y. Zhong, and Z. Huang, "An improved blind recognition method of the convolutional interleaver parameters in a noisy channel," IEEE Access, vol. 7, pp. 101775-101784, 2019. doi: 10.1109/ACCESS.2019.2930663.

[50] F. C. P. Barros, M. L. M. G. Alcoforado, and W. T. A. Lopes, "Efeito do desvanecimento Rayleigh no desempenho dos códigos polares," in Anais da IX Conferência Nacional em Comunicações, Redes e Segurança da Informação (Encom 2019), Petrolina, PE, Outubro 2019, pp. 87-88.

[51] X. Fan, P. Bai, X. Liang, J. Zhang, and B. Liu, "Detection algorithm of BPSK signal of parameter-adjusted bistable stochastic resonance model based on scale change," IEEE Access, vol. 8, pp. 97643-97 657, 2020. doi: 10.1109/ACCESS.2020.2997705.

[52] M. S. Omeira, G. M. Hamad, and A. D. Elbayoumy, "A code-matched collision-free S-random interleaver for turbo codes," in 2015 IEEE Seventh Int. Conf. on Intelligent Computing and Information Systems (ICICIS), 2015, pp. 398-404. doi: 10.1109/IntelCIS.2015.7397251.

[53] J. S. Yedidia, W. T. Freeman, Y. Weiss et al., "Understanding belief propagation and its generalizations," Exploring Artificial Intelligence in the New Millennium, vol. 8, pp. 236-239, 2003.

[54] C. Huang, H. Wang, C. Peng, C. Tsai, C. Wu, and G. Lin, "Multimode VCSEL enables 42-GBaud PAM-4 and 35-GBaud 16-QAM OFDM for 100-m OM5 MMF Data Link,” IEEE Access, vol. 8, pp. 36963-36973, 2020. doi: 10.1109/ACCESS.2020.2975127.

[55] Y. Fu, C. Wang, X. Fang, L. Yan, and S. Mclaughlin, "BER performance of spatial modulation systems under a non-stationary massive MIMO channel model," IEEE Access, vol. 8, pp. 44547-44558, 2020. doi: 10.1109/ACCESS.2020.2977139.

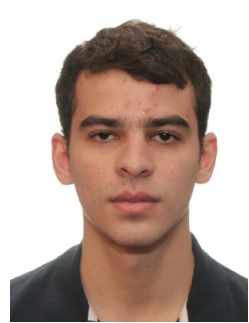

Francisco Caio Parente de Barros was born in Araripina, Pernambuco, Brazil on May 25th, 1993. $\mathrm{He}$ received his BSc. in Electrical Engineering from State University of Pernambuco in 2016 where he also received his M.Sc. degree in Systems Engineering in 2021. His research interests include polar codes, information theory, digital signal processing and coding. 


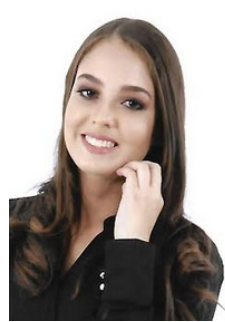

Flávia Camila Morais de Oliveira was born in Recife, Pernambuco, Brazil on December 7th, 1995. She recieved the B.Sc. degrees in Electrical Engineering from State University of Pernambuco, Brazil, in 2018, where she also received her M.Sc. Degrees in Systems Engineering in 2021. Her research interests include polar codes, modulation, coding theory and digital signal processing.

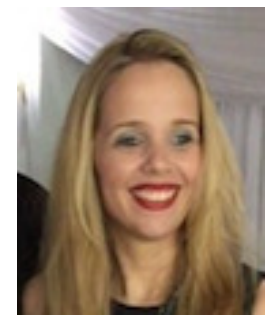

Maria de Lourdes Melo Guedes Alcoforado received her B.Sc. degree in electrical/Electronics Engineering (1995), the M.Sc. (2000) and D.Sc. degrees (2005) in Electrical Engineering from the Federal University of Pernambuco, Brazil. She was a researcher (2013-2016) in the project entitled Coding and Security Advanced Techniques for Wireless Communications, involving the School of Computing and Communications, Lancaster University and the Communications Research Group, Federal University of Pernambuco. Since 2001 she is with the University of Pernambuco, dedicated to researching and teaching in Undergraduate and Post Graduate courses. She was the Coordinator of the Post Graduate Program in Systems Engineering (2016-2021) and currently she is a Sector Coordinator for Post Graduate Studies and Research at the Polytechnic School of Pernambuco. Her research interests include polar codes, turbo codes, multiple access channel, modulation and cryptography.

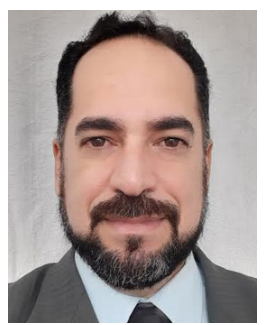

Waslon Terllizzie Araújo Lopes was born in Petrolina, Pernambuco, Brazil on December, 29, 1974. He received the B.Sc. and M.Sc. degrees in Electrical Engineering from Federal University of Paraiba, Brazil, in 1998 and 1999, respectively. He received his D.Sc. degree in Electrical Engineering from Federal University of Campina Grande, Brazil in 2003. From August 2003 to December 2009 he was with ÁREA1 College of Science and Technology in Salvador, Brazil, where he was the head of the Telecommunications Group. From December 2009 to April 2015 he was with Department of Electrical Engineering of Federal University of Campina Grande, Brazil. From November 2018 to October 2019 he was visiting professor in the University of Toronto, Canada. Currently, Dr. Waslon is Associate Professor in the Department of Electrical Engineering of the Center of Alternative and Renewable Energy of Federal University of Paraíba, Brazil. He is also Financial Director of the Institute for Advanced Studies in Communications (Iecom). His research interests include robust vector quantization, wireless communication systems, communication theory, and digital signal processing. Dr. Waslon co-authored the books "Communications, Information and Network Security" published by Kluwer Academic Publishers and "Spectral Sensing: Techniques and Applications" published by Momentum Press. Waslon Terllizzie is member of the Brazilian Telecommunications Society (SBrT) since 1997 and Senior Member of the Institute of Electrical and Electronics Engineers (IEEE). 